\title{
Strategic Research on Carrying Forward an Innovative Health Service System for Traditional Chinese Medicine
}

\author{
Dang Haixia ${ }^{1}$, Zhang Junhua ${ }^{2}$, Liu Baoyan ${ }^{1}$, Su Qinmin ${ }^{1}$, Xiao Mengxiong ${ }^{1}$, Yang Yongshen ${ }^{1}$, Zhang Zedong ${ }^{1}$, \\ Zhang Boli ${ }^{1}$
}

1. China Academy of Chinese Medical Science, Beijing 100700, China

2. Tianjing University of Traditional Chinese Medicine, Tianjin 300193, China

\begin{abstract}
Based on literature analysis, expert interviews, and field investigation, this paper systematically analyzed the current situation and problems in the development of Chinese medicine health services from pre-disease to after-disease. Moreover, policy recommendations were put forward, including establishing a management system suitable for the development of traditional Chinese medicine (TCM) to bring the health care work of TCM forward, improving the standards and management system of Chinese medicine health services, broadening the service field, and innovating service models to promote the development of TCM health services, and promoting the globalization of TCM to propagate traditional Chinese culture.
\end{abstract}

Keywords: traditional Chinese medicine; health service system; inheritance; innovation; development strategy

\section{Introduction}

With the rapid development of the social economy, the aging population, and the blowout of chronic disease, one of the most imperative demands is to improve the level of Chinese inhabitants' health. Improving and promoting the health of the people of China and Chinese health constructs have become national strategies in the new era. Additionally, developing a healthy industry has been a novel economic growth point. Traditional medicine, while adding uniqueness to the Chinese medical system, also competes mutually and develops harmoniously with Western medicine. Meanwhile, they jointly undertake a mission to maintain and enhance people's health. The state department issued the Chinese Medicine Development Strategy Plan (20162030) on February 26, 2016 and moved on further to identify the developing orientation and key work of the next fifty years. Xi Jinping, the president of China, signed the Chairman Order
No. 59 on December 25, 2016. The National People's Congress Standing Committee testified and published the Law of People's Republic of China on Traditional Chinese Medicine. In previous eras, Chinese medicine received unprecedented attention. There were speedy developments such as increasing medical apparatuses, expanding the team of geniuses, and enhancing their service skills. In 2014, there were 43586 domestic medical institutions accounting for $4.4 \%$ of the whole medical and health institutions (Fig. 1); with the total number of visits increasing from 613 million in 2010 to 874 million in 2014 an average annual growth rate of $9.3 \%$ [1]. In particular, according to statistics of the World Federation of Acupuncture-Moxibustion Societies in 2015, a total of 183 countries and regions have opened clinics related to Chinese medicine. Chinese medicine has become the second largest medical system in the world comparable to Western medicine; solving many clinical problems which are difficult to solve by Western medicine and thus, playing an irreplaceable

Received date: December 16, 2016; Revised date: March 20, 2017

Corresponding author: Zhang Boli, Tianjin University of Traditional Chinese Medicine, China Academy of Chinese Medical Sciences, Professor; Chinese Academy of Engineering, Academician. Major research fields include heart and brain vascular disease. E-mail: zhangbolipr@163.com

Funding program: CAE Advisory Project “Development Strategy for National Health Promotion and Medical and Healthcare Undertakings in China” (2014-ZD-06)

Chinese version: Zhang Boli et al. Strategic research on carrying forward an innovative health service system for Traditional Chinese Medicine. Strategic Study of CAE, https://doi.org/10.15302/J-SSCAE-2017.02.014 
role in health care. It will soon become an indispensable contributor to the future development of medicine. However, the construction of TCM health service system is still facing a severe situation. Chinese medicine tends to overlook Westernization; hence, the role of its advantages is blocked, and the pressure of inheritance is enormous. The concept of TCM "preventive treatment of disease" has failed to be effectively integrated into the promotion of universal health, and its contribution to health has not been highlighted. Therefore, based on a complete statistical analysis of TCM from 2010 to 2014, interviews were conducted with more than 100 experts and managers, and a thorough investigation of Gansu, Shaanxi, Sichuan, Guangzhou, Shandong, Jiangxi, Jiangsu, and Fujian provinces and cities occurred. The advantages and existing problems in the development of TCM health services from pre-illness, disease, and post-illness were analyzed to seek a breakthrough in the health services of TCM. The analysis was also carried out to build on TCM innovation and health service development strategies of the system.

\section{Formulating measures and promoting implementation to consolidate the policy system of TCM policy}

An idea is clearly proposed to develop modern medicine and traditional medicine in the Constitution of the People's Republic of China. However, combining TCM with Western medicine has always been the basic policy of China's health issues. In recent years, the state's financial allocations to Chinese medical institutions have been increasing every year, from 14.474 billion yuan in 2010 to 23.769 billion yuan in 2014, alongside financial inputs. However, the proportion of financial allocations to Chinese medical and health institutions has been declining (Table 1) [1]. The Chinese medicine management system still reflects a situation which is vertically "high paraplegia" and horizontally "multi-sectoral management." It is difficult to form a concerted effort as its resources are scattered. Therefore, all levels of government should ensure a steady investment in medicine and taxation of TCM to encourage the development of both Chinese and Western medicine, strengthen the system reform of Chinese medicine, unify planning and leadership, and finally, highlight the role of Chinese medicine in the medical and health system. These measures are aimed to ensure better implementation of national policies, and to strengthen the Chinese Academy of Traditional Chinese Medicine's overall planning in the scientific research system, resource integration, and coordinating role as an organization. There is a need to establish a scientific and technological organization similar to the National Institutes of Health (NIH) to give full play to the advantages of the system and speed up the development of the scientific research innovation system of Chinese medicine. In deepening the reform of the pharmaceutical industry, we will further improve and implement the relevant policies of Chinese medicine and formulate favorable policies that will benefit the development of the Chinese medicine health service industry.

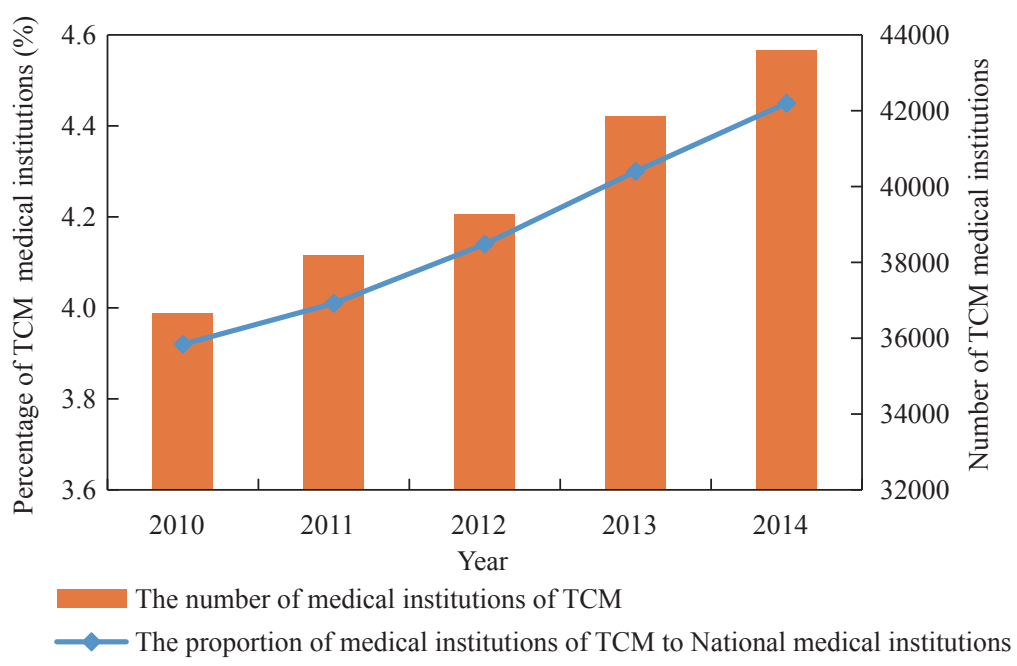

Fig. 1. The number and proportion of TCM medical institutions from 2010 to 2014.

Table 1. The percentage and total amount of financial appropriation to medical institutions of TCM [1].

\begin{tabular}{|c|c|c|c|c|c|}
\hline Item & 2010 & 2011 & 2012 & 2013 & 2014 \\
\hline Medical institution (100 million yuan) & 2091.24 & 2781.34 & 3120.51 & 3468.12 & 4646.39 \\
\hline Medical institution of TCM (100 million yuan) & 144.74 & 168.98 & 172.68 & 192.08 & 237.69 \\
\hline Percentage $(\%)$ & 6.92 & 6.08 & 5.53 & 5.54 & 5.12 \\
\hline
\end{tabular}




\section{Strengthening primary care and improving service ability to perfect the service network of "preventative treatment of disease"}

"Preventive treatment of disease" emphasizes the early diagnosis of disease and intervention. Meanwhile, it also reflects the concept of preventing and controlling a disease from developing into a serious illness. By using the human senses, Chinese medicine practitioners can diagnose and conduct interventions with a person during the quantitative phase of their state of health by looking, smelling, asking, and cutting through their external manifestations, which have simple, convenient, inexpensive, effective, and safe comprehensive advantages. "Preventive treatment of disease" in line with the current concept of sanitary and healthful view, is beneficial for Chinese medicine health service to guide and stimulate demand. The National Health and Wellness Conference held on August 20, 2016, clearly pointed out the present health policy which includes "taking the grassroots" as the focal point, perceiving "reform and innovation" as the driving force, seeing "prevention" as the mainstay, combining Chinese and Western medicine, and integrating health into all policies that are co-constructed and shared amongst all people [2]. However, a long-standing emphasis on disease treatment and neglect of pre-disease prevention has led to rehabilitation after treatment. These outcomes have restricted the full play to the traditional advantages of the TCM's' "preventive treatment of disease". Therefore, it has not yet formed a systematic, standardized, and efficient "preventive treatment of disease" service network. The grassroots medical institutions closest to the masses are the cornerstone of Chinese medicine's "preventive treatment of disease". Only if we do a good job at the grassroots level can we "cure diseases without disease" and effectively achieve health care work that is at a "pass" level. In 2015, the number of practicing (assistant) physicians in the Chinese medicine category accounted for $20.11 \%, 15.59 \%$ and $16.9 \%$ respectively of the community health service centers (stations), township hospitals, and village clinics [1]. At the grassroots level, there is a lack of qualified Chinese medicine practitioners and has a long way to form a strong base for Chinese medicine services. Therefore, the Chinese medicine "preventive treatment of disease" service is an essential part of the national basic public health service project. China should: (1) take community and rural areas as important platforms to implement "preventive treatment of disease" services; (2) take TCM, acupuncture, massage, and other non-drug therapies as a comprehensive treatment of primary health care institutions during basic diagnosis and treatment; (3) establish a "Chinese medicine museum" cloud platform and a national "preventive treatment of disease" information network in grassroot medical institutions to enhance the level of "preventive treatment of disease" within primary medical institutions; (4) strengthen the cultivation and introduction of TCM talents at grassroots level; (5) establish a disciplinary system for the prevention and treatment of diseases, and training talents; and (6) guide the advantageous resources of TCM to start at a grassroot level of treatment and provide basic support for effective implementation of "preventive treatment of disease."

\section{Determining the advantages, grasping the key points, and seeking breakthroughs to highlight the contribution of TCM}

Although the indispensable role of Chinese medicine in the universal health system has been widely recognized, the total amount of Chinese medicine resources is still inadequate. The appropriate technology of TCM has not been fully popularized and applied, and the prices of its technical services are so low that some special technology used at clinics is on the verge of loss. The contribution of TCM to health has not been effectively highlighted. In this regard, the advantages of TCM should be cleared and emphasized during breakthrough in pre-illness, disease, and post-illness at different stages. We will intensify the research and promotion of non-medical Chinese medicine in the field of health services such as "preventive treatment of disease," and chronic disease prevention and rehabilitation. Integrated resources and focus on cancer, cardiovascular and cerebrovascular diseases, metabolic diseases, new infectious diseases, and other major diseases, give full play to the advantages of TCM. Carrying out joint research on the key links between basic research, clinical evaluation, and mechanism of action, helps to identify the advantages of Chinese medicine and strive for a breakthrough. Effectively and vigorously integrating TCM ancient literature with modern science and technology, promotes the theoretical innovation of TCM. It is important to establish a chronic disease prevention and treatment system featuring TCM, integrating traditional Chinese with Western medicine to further tap the potential of TCM in the prevention and control of chronic diseases, promote the effective combination of TCM with Western medicine, and conduct and improve the division of labor between Chinese medicine hospitals and primary health care institutions. These would also include disease prevention and control institutions to cooperate in the prevention and treatment of chronic diseases; forming a working mechanism to combine the comprehensive prevention and treatment of chronic diseases with clinical research. Additionally, it would give full play to the obvious advantages of TCM in pediatrics, gynecology, and reproductive diseases, and revitalize the pediatrics of TCM and speed up the restoration of pediatrics in hospitals more than Grade II. In the fields of gynecology and reproduction, we will facilitate the advantages of integration of traditional Chinese and Western medicine and closely integrate the overall regulation of Chinese medicine with the precision of Western medicine. 


\section{Improving the standards and management system of TCM hierarchically and highlighting its characteristics}

At present, China has not yet established the methods and models which have led to the formulation of Chinese medicine standards. The mode of individualized diagnosis and treatment of Chinese medicine have not been effectively linked with the standardized method system. The standard system of Chinese medicine is not perfect, and its authority and recognition are not sufficient. It also lacks support for career development. With the vigorous development of TCM and health services, the construction of the Chinese medicine standard system (including technology, service, personnel) has become top priority; we must promote the construction of a standardized support system for Chinese medicine. Correspondingly, we must actively promote the standardization of TCM diagnosis and treatment technology to: improve the quality and effectiveness of medical services; establish the Chinese medicine evaluation standard system focusing on strengthening the efficacy evaluation of Chinese medicine, personnel evaluation, evaluation of scientific research, and professional title evaluation standard system; promote the development of international standards for Chinese medicine; strengthen research on chemical contamination and heavy metal standards of Chinese herbal medicines; improve the quality of Chinese herbal medicines; standardize the "preventive treatment of disease" industry standards; and establish characteristics consistent with the TCM system.

\section{Expanding the medicine service field, increasing supply, and creating a new model to make the TCM health service industry bigger and stronger}

The health industry is a new industry with a huge potential market. In China, the integration of Chinese medicine and the health industry can broaden the field of Chinese medicine service, increase and improve the quality of the supply of health services in China, and create a health service model with Chinese characteristics to form a large-scale and comprehensive Chinese medicine health service industry. Therefore, it is imperative to promote the complete integration and development of TCM services, health care meals, the idea of "preventive treatment of disease," acupuncture and massage, Taijiquan, and Baduanjin for the normalization, standardization, and modernization of health care and pension industries. In view of the shortage of hospital beds in China and the lack of medical services for discharged patients, we should take full advantage of the role of TCM in disease rehabilitation, and establish and optimize a comprehensive system combining TCM with Western medicine; linking rehabilitation with regimen and leisure. Finally, we must effectively reduce hospital pressure to extend the chain of medical services, vigorously promote the development of non-public medical institutions, and encourage more practice of Chinese medicine practitioners. We will make full use of information technologies such as the Internet and big data to promote the innovation of service models such as smart medicine and smart healthcare in TCM, so as to integrate Chinese medicine into all fields related to health services.

\section{Carrying forward the excellent Chinese culture and promoting the development of Chinese medicine overseas}

The World Health Organization (WHO) report shows that more than 100 million Europeans with a ratio of 1 in 5 persons, are currently users of traditional and complementary alternative medicine. In Japan, $84 \%$ of Japanese doctors use Kampo medicine in their daily practice. Acupuncture has been approved and used in 103 member states of the WHO [3]. According to incomplete statistics, from 1987 to 2005 , China trained more than 54700 people studying Chinese medicine in more than 130 countries and regions [4]. About 13 of the total of 15 traditional medicine collaborating centers in Asia established by the WHO, 7 of which are in China, are related to TCM. Acupuncture as a theory under the guidance of Chinese medicine has been spread in 183 countries and regions. However, Chinese medicine has not yet entered the mainstream medical system. Chinese medicine is a significant carrier of China's outstanding traditional culture. Promoting the expansion of Chinese medicine services overseas is not only limited to residents in other countries but can also effectively enhance China's cultural soft power. At present, we should set up several "one belt, one road" Chinese medicine centers overseas by relying on backbone organizations for international exchange and cooperation in medical, education, and scientific research. We will improve the hardware environment, build a program that can undertake intergovernmental cooperation projects, and demonstrate the modernization and internationalization of Chinese medicine. We will also accelerate the international dissemination of Chinese medicine culture, the construction of international standards for Chinese medicine, and cultivate a group of Chinese medicine service enterprises or enterprise groups with strong international market development capability to promote Chinese medicine abroad and establish a foothold globally.

\section{References}

[1] Department of Financial and Planning of State Administration of Traditional Chinese Medicine of the People's Republic of China. Statistics compile of TCM (1995-2015). http://www.satcm.gov. cn/2015tjzb/start.htm. Chinese

[2] Learning China. Present Xi Jinping proposed the new approach 
of health and care. http://www.ccln.gov.cn/xxzgyc/200201.shtml. Chinese.

[3] World Health Organization. WHO traditional medicine strategy 2014-2023 [M]. [2016-11-15]. http://www.who.int/publications/ list/traditional_medicine_strategy/zh/. Chinese.

[4] Liu M. Research on education of foreign students of TCM [D]. Changchun: Northeast Normal University (Master's thesis), 2007. Chinese. 